\title{
Is intraoperative ultrasound (IOUS) still useful for the detection of liver metastases?
}

\author{
M. D’Onofrio ${ }^{\text {a,*}}$, A. Gallotti ${ }^{a}$, E. Martone ${ }^{a}$, L. Nicoli ${ }^{\text {a }}$, S. Mautone ${ }^{\text {a }}$, \\ A. Ruzzenente ${ }^{b}$, R. Pozzi Mucelli ${ }^{a}$ \\ a Department of Radiology, Policlinico Universitario G.B. Rossi, University of Verona, Italy \\ b Department of Surgery and Gastroenterology, Policlinico Universitario G.B. Rossi, University of Verona, Italy
}

\author{
KEYWORDS \\ Liver; \\ Metastases; \\ Ultrasound; \\ Computed tomography; \\ Magnetic resonance; \\ Intraoperative \\ ultrasound.
}

\begin{abstract}
Purpose: To evaluate the clinical impact of intraoperative ultrasound (IOUS) in the detection of liver metastases during the years, as compared with those of other imaging modalities. Materials and methods: All IOUS scans performed for detection of liver metastases from 2000 to 2006 were retrospectively reviewed and compared with the results of preoperative imaging modalities: Ultrasound (US), Computed Tomography (CT), and/or Magnetic Resonance (MR). The number of cases in which IOUS and preoperative imaging studies produced discordant results, in terms of presence/absence of focal liver lesions, was calculated per year. Statistical analysis was performed using the McNemar test. A $p$ value $<0.05$ was considered statistically significant.

Results: Eighty-three IOUS scans performed in 2000-2003 were reviewed, and discordance with preoperative imaging findings was found in 19/83 (23\%) cases. Of the 42 IOUS scans done during the 2004-2006 period, 10/42 (24\%) showed discordance with preoperative studies. All metastases diagnosed with imaging studies were pathologically confirmed. The number of discordant cases in the two periods were not significantly different $(p=0.2)$.

Conclusion: IOUS is still useful in the detection of liver metastases. Its decreased use is probably due to the improved accuracy of preoperative imaging modalities.

Sommario Scopo: Valutare l'impatto clinico negli anni dell'ecografia intraoperatoria (IOUS) nella identificazione delle metastasi epatiche rispetto alle altre metodiche di imaging.

Materiali e metodi: risultati delle ecografie intraoperatorie effettuate per l'identificazione delle metastasi epatiche in un periodo compreso tra gli anni 2000 e 2006 sono stati retrospettivamente revisionati e comparati con le metodiche di imaging preoperatorio: Ecografia (US), Tomografia Computerizzata (TC) e/o Risonanza Magnetica (RM). Tutti i casi sono stati patologicamente provati. Sono stati calcolati per anno i casi discordanti in termini di presenza/assenza di lesione focale epatica. Il confronto statistico è stato effettuato con il test di McNemar. Un valore del $p<0,05$ è stato considerato statisticamente significativo.
\end{abstract}

\footnotetext{
* Corresponding author. Istituto di Radiologia, Policlinico Universitario G.B. Rossi, Università di Verona, P.le L. A. Scuro 10, 37134 Verona, Italy.

E-mail address: mirko.donofrio@univr.it (M. D’Onofrio).
} 
Risultati: Nel triennio 2000-2003, i dati di 83 pazienti sono stati revisionati e i casi discordanti sono stati $19 / 83$ (23\%). Nel triennio 2004-2006, i dati di 42 pazienti sono stati revisionati e $i$ casi discordanti sono stati $10 / 42(24 \%)$. Non vi è stata differenza statisticamente significativa $(p=0,2)$ circa il numero di casi discordanti nei due periodi. L'esame patologico ha confermato la presenza di metastasi in tutti i casi.

Conclusioni: L'ecografia intraoperatoria (IOUS) risulta una metodica di imaging tuttora utile nella identificazione delle metastasi epatiche. La riduzione del suo impiego negli anni è probabilmente dovuta alla maggiore accuratezza diagnostica dell'imaging preoperatorio.

(c) 2009 Elsevier Srl. All rights reserved.

\section{Introduction}

Since the 1980s, various groups have documented the usefulness of intraoperative ultrasonography (IOUS) in hepatobiliary and pancreatic surgery [1-3]. Knight and Newell described IOUS for the first time in 1963 and showed that A-mode scans could be used to demonstrate the presence of stones in the gallbladder and common bile duct [4]. Technical developments improved the diagnostic accuracy of IOUS and rendered it increasingly useful for correcting and refining therapeutic decisions [5-8]. It has also proved to be valuable in guiding surgical procedures $[5,9]$, particularly minimally invasive laparoscopic techniques, in which direct visual and manual inspection of the affected organs and the retroperitoneum is not possible. However, the role of IOUS has recently been downsized, especially in hospitals where advanced imaging techniques are used preoperatively [10]. The aim of this study was to evaluate the clinical impact of IOUS in the detection of focal liver lesions over the years, comparing it with other imaging modalities.

\section{Materials and methods}

We retrospectively assessed all hepatic IOUS scans performed in our institute on patients with colon cancer between 2000 and 2006 and compared the findings with those of preoperative imaging studies, including Ultrasound (US), Computed Tomography (CT), and/or Magnetic Resonance (MR). Patients with incomplete preoperative imaging studies were excluded.

\section{IOUS examination protocol}

We used dedicated, sterilized, high-frequency I-shaped or T-shaped transducers (Fig. 1). Both liver lobes were scanned at frequencies ranging from 3 to $13 \mathrm{MHz}$. Lower frequency $(3-7 \mathrm{MHz})$ was used to examine the posterior aspect of the left lobe with the transducer positioned on the anterior surface of the liver. The posterior segments of the right lobe were studied by direct application of the transducer on the posterior surface of the liver. If palpation revealed suspicious superficial nodules, the liver surface was explored with a high-frequency $(7-13 \mathrm{MHz})$ transducer $[5,6]$. Each new lesion discovered in this manner was subjected to cytological examination with immediate response.

\section{Data analysis}

All IOUS examinations were retrospectively reviewed and compared with the results of preoperative imaging studies (US, CT, and/or MRI). The number of discordant cases in terms of presence/absence of focal liver lesions were calculated per year. Statistical analysis was based on the

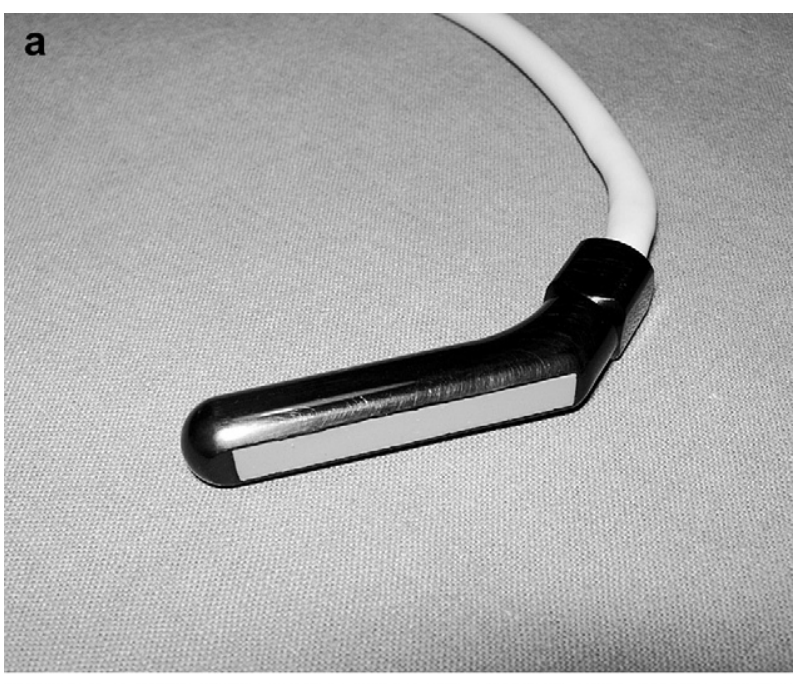

b

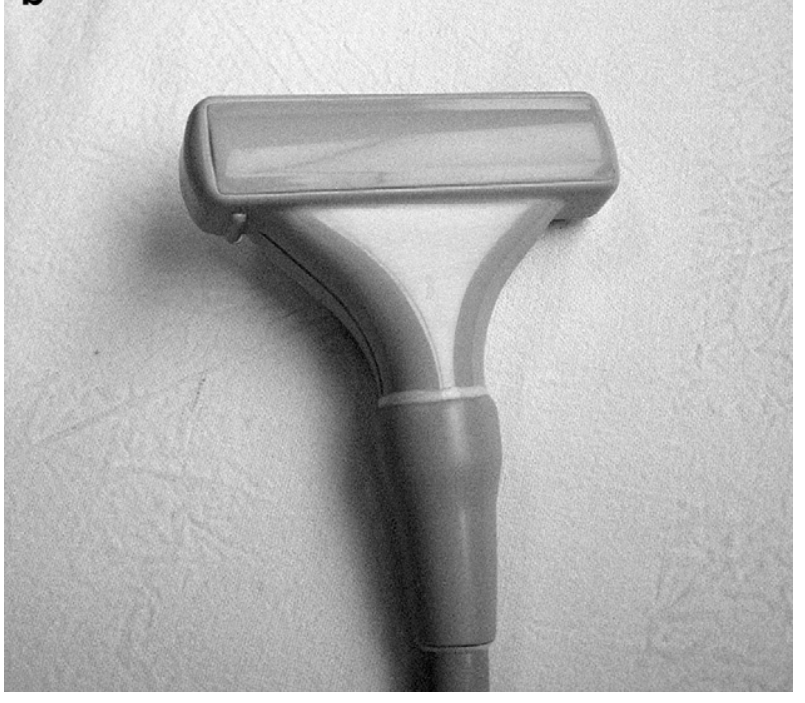

Fig. 1 Dedicated (a) I-shaped and (b) T-shaped intraoperative ultrasound transducers. 
McNemar test. A $p$ value $<0.05$ was considered statistically significant.

\section{Results}

Our analysis included 83 patients who underwent IOUS between 2000 and 2003 and 42 who were studied between 2004 and 2006. Discordance between IOUS findings and the results of preoperative imaging studies was found in 19 of the $83(23 \%)$ patients examined during the first period and 10/42 (24\%) of those examined later. The rates of discordance in the two periods were not significantly different $(p=0.2)$.

The discordant cases (Fig. 2) included 29 unexpected focal liver lesions (maximum diameter $5-20 \mathrm{~mm}$ ) detected at IOUS. The metastases were located in the right lobe in 15/29 (52\%) cases, in the left lobe in $12 / 29(41 \%)$, and in the caudate lobe in $1 / 29(3 \%)$. In the remaining $2 / 29$ (7\%) cases, there was diffuse metastatic involvement of the liver surface. All 29 were pathologically diagnosed as metastatic lesions.

\section{Discussion}

Identification of metastatic involvement is an important part of liver staging, so accurate exploration of the
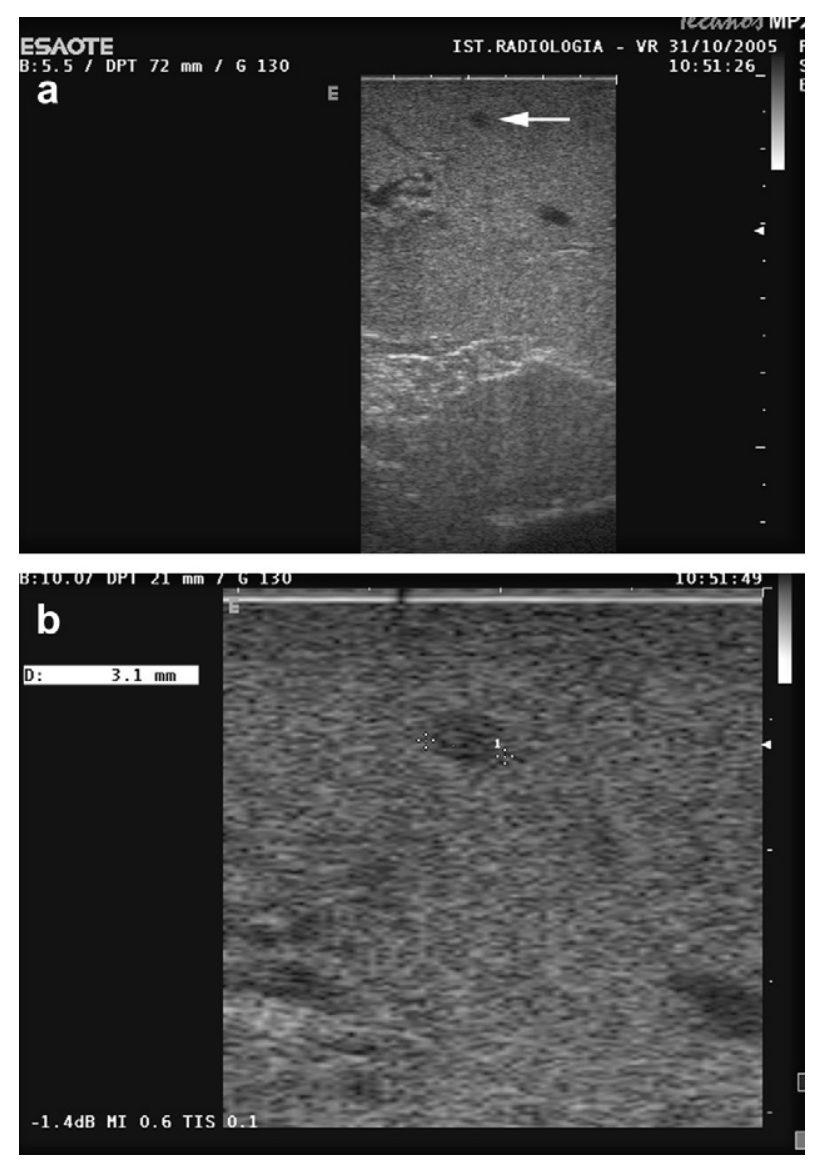

Fig. 2 IOUS reveals a small focal liver metastases missed on preoperative imaging studies. (a) A tiny hypoechoic focal liver lesion measuring $3 \mathrm{~mm}$ in diameter. (b) The lesion was not detected at pre-surgical imaging studies and then identified at IOUS. entire parenchyma is compulsory. High-resolution IOUS can detect very small hepatic lesions (including those that are marginally located), which are often undetectable on preoperative imaging studies, and its use increases the duration of surgery by no more than 1015 minutes [5].

Several studies showed the efficacy of IOUS in detecting small liver lesions: the examination led to new diagnoses in $30 \%$ of patients with HCCs in cirrhotic livers with hard, irregular surfaces [11] and in $10 \%-40 \%$ of those with metastases from colorectal cancer [12]. However, other authors who compared IOUS with preoperative imaging studies reported that the intraoperative examination had an impact on decision making in only $4 \%-7 \%$ of all cases $[13,14]$, depending on the surgical strategy of each team and the tumor type. If the patient is scheduled to undergo major hepatectomy, the intraoperative detection of one or more additional lesions in the same lobe will not affect the treatment plan, but it may have a greater impact if a parenchyma-sparing resection has been planned.

Compared with CT and MRI, IOUS was found to play a primary role in tumor staging by Zacherl et al., who found that it provided new treatment-relevant information on the location, number, and topographic features of liver metastases in $29.5 \%$ of cases [15]. In 2004, however, Sahani et al. reported the advantages of IOUS with respect to contrast-enhanced MR using a hepatospecific contrast medium. The sensitivity of IOUS and MR was $94.3 \%$ and $86.7 \%$, respectively [10].

Guimaraes et al. showed that IOUS plays a central role in the detection of focal liver lesions in patients undergoing laparotomy for abdominal tumors [7]. They compared preoperative imaging studies (US and CT) with intraoperative inspection, palpation, and IOUS. The histopathological data confirmed the higher diagnostic accuracy of IOUS in detecting hepatic lesions that had not been identified preoperatively. Moreover, IOUS provides surgeons with important information on the consistency and vascular features of the lesions and also reveals anatomic variations $[7,16]$

IOUS has also been used successfully to guide biopsies, fine-needle aspirations $[17,18]$ and therapeutic procedures, such as thermoablation, cryoablation, or alcohol injections $[5,19]$.

In recent years, the role of IOUS has been downsized as a result of improvements in techniques used for preoperative imaging (e.g., technical innovations, the development of new contrast agents) $[18,20,21]$. The use of contrast agents during IOUS has also been reported to increase its diagnostic accuracy and improve its ability to detect and characterize focal liver lesions [18,21-24]. With respect to contrast-enhanced IOUS studies performed with convex transducers, the use of dedicated probes should provide better results.

In conclusion, although improved preoperative imaging has lessened the need for IOUS, our study confirms that this approach still offers excellent accuracy. Contrast-enhanced IOUS should play an important role in the detection and characterization of hidden focal liver lesions. The most important limitation of the present study is its retrospective design. 


\section{Conflict of interest statement}

The authors have no conflict of interest.

\section{References}

[1] Clarke MP, Kane RA, Steele Jr G, Hamilton ES, Ravikumar TS, Onik G, et al. Prospective comparison of preoperative imaging and intraoperative ultrasonography in the detection of liver tumors. Surgery 1989;106:849-55.

[2] Smith SJ, Vogelzang RL, Donovan J, Atlas SW, Gore RM, Neiman HL. Intraoperative sonography of the pancreas. AJR Am J Roentgenol 1985 Mar;144(3):557-62.

[3] Onik G, Kane R, Steele G, McDermott W, Khettry U, Cady B, et al. Society of Gastrointestinal Radiologists Roscoe E. Miller Award. Monitoring hepatic cryosurgery with sonography. AJR Am J Roentgenol 1986;147:665-9.

[4] Knight PR, Newell JA. Operative use of ultrasonics in cholelithiasis. Lancet 1963;1:1023-5.

[5] Machi J, Oishi AJ, Furumoto NL, Oishi RH. Intraoperative ultrasound. Surg Clin North Am 2004;84:1085-111.

[6] Long EE, Van Dam J, Weinstein S, Jeffrey B, Desser T, Norton JA. Computed tomography, endoscopic, laparoscopic, and intra-operative sonography for assessing resectability of pancreatic cancer. Surg Oncol 2005;14:105-13.

[7] Guimarães CM, Correia MM, Baldisserotto $M$, de Queiroz Aires EP, Coelho JF. Intraoperative ultrasonography of the liver in patients with abdominal tumors: a new approach. $J$ Ultrasound Med 2004;23:1549-55.

[8] Solomon MJ, Stephen MS, Gallinger S, White GH. Does intraoperative hepatic ultrasonography change surgical decision making during liver resection? Am J Surg 1994;168:307-10.

[9] Ozsunar Y, Skjoldbye B, Court-Payen M, Karstrup S, Burcharth F. Impact of intraoperative ultrasonography on surgical treatment of liver tumors. Acta Radiol 2000;41:97-101.

[10] Sahani DV, Kalva SP, Tanabe KK, Hayat SM, O’Neill MJ, Halpern EF, et al. Intraoperative US in patients undergoing surgery for liver neoplasms: comparison with MR imaging. Radiology 2004;232:810-4.

[11] Kokudo N, Bandai Y, Imanishi H, Minagawa M, Uedera $Y$, Harihara Y. Management of new hepatic nodules detected by intraoperative ultrasonography during hepatic resection for hepatocellular carcinoma. Surgery 1996;119(6):634-40.
[12] Machi J, Isomoto H, Kurohiji T. Accuracy of intraoperative ultrasonography in diagnosing liver metastasis from colorectal cancer: evaluation with postoperative follow-up results. World J Surg 1991;15:551-6.

[13] Jarnagin WR, Bach AM, Winston CB, Hann LE, Heffernan N, Loumeau $\mathrm{T}$, et al. What is the yield of intraoperative ultrasonography during partial hepatectomy for malignant disease? J Am Coll Surg 2001;192(5):577-83.

[14] Cerwenka H, Raith J, Bacher H, Werkgartner G, el-Shabrawi A, Kornprat $\mathrm{P}$, et al. Is intraoperative ultrasonography during partial hepatectomy still necessary in the age of magnetic resonance imaging? Hepatogastroenterology 2003;50(53): 1539-41.

[15] Zacherl J, Scheuba C, Imhof M, Zacherl M, Längle F, Pokieser P, et al. Current value of intraoperative sonography during surgery for hepatic neoplasms. World J Surg 2002;26:550-4.

[16] Torzilli G, Montorsi M, Del Fabbro D, Palmisano A, Donadon M, Makuuchi M. Ultrasonographically guided surgical approach to liver tumours involving the hepatic veins close to the caval confluence. Br J Surg 2006;93(10):1238-46.

[17] Martínez-Noguera A, Montserrat E, Torrubia S, Monill JM, Estrada P. Ultrasound of the pancreas: update and controversies. Eur Radiol 2001;11:1594-606.

[18] Kokudo N, Imamura H, Sano K, Zhang K, Hasegawa K, Sugawara $Y$, et al. Ultrasonically assisted retrohepatic dissection for a liver hanging maneuver. Ann Surg 2005;242:651-4.

[19] Gaitini D, Kopelman D, Soudak M, Epelman M, Assalia A, Hashmonai $M$, et al. Impact of intraoperative sonography on resection and cryoablation of liver tumors. J Clin Ultrasound 2001;29:265-72.

[20] Solbiati L, Tonolini M, Cova L, Goldberg SN. The role of contrast-enhanced ultrasound in the detection of focal liver lesions. Eur Radiol 2001;11:E15-26.

[21] Siosteen AK, Elvin A. Intra-operative uses of contrastenhanced ultrasound. Eur Radiol 2004;14:P87-95.

[22] Hiramoto JS, Feldstein VA, LaBerge JM, Norton JA. Intraoperative ultrasound and preoperative localization detects all occult insulinomas; discussion 1025-6. Arch Surg 2001;136: 1020-5.

[23] D’Onofrio M, Vecchiato F, Faccioli N, Falconi M, Pozzi Mucelli R, et al. Ultrasonography of the pancreas. 7. Intraoperative imaging. Abdom Imaging 2007;32(2):200-6.

[24] Torzilli G. Contrast-enhanced intraoperative ultrasonography in surgery for liver tumors. Eur J Radiol 2004;51(suppl. 1): S25-9, Review. 